\title{
Effect of fermented kitchen waste on tilapia (Oreochromis niloticus) growth performance and water quality as a water additive
}

\begin{abstract}
Aquaculture contributes about $20 \%$ of domestic fish production in Malaysia. Tilapia has been identified as one of the main species for freshwater aquaculture in the Third National Agriculture Policy (DPN3). However, feed cost and water quality management remain as two major challenges to the industry. This study aim to analyse the effects of Fermented Kitchen Waste (FKW) as water additives on water quality and growth performance of tilapia (Oreochromis niloticus). Different concentration $(0.05,0.1$ and $0.2 \%)$ of FKW were used to treat tilapia in tank culture for a period of twelve weeks. Physico-chemical parameters were also taken every week. Treatment with $0.1 \%$ FKW resulted in significant $(\mathrm{p}<0.05)$ decrease in ammonia, nitrite and nitrate levels. The survival rates of tilapia treated with 0.05 and $0.1 \%$ FKW were comparable to the untreated control. Growth performance of the tilapia was measured in term of length and weight. Highest relative growth rate was observed in tilapia treated with $0.05 \%$ FKW. However, all the fish died in $0.2 \%$ FKW due to severe pH drop. Therefore, low concentration of FKW could severe as a potential water additive to improve water quality and promote growth in tilapia aquaculture.
\end{abstract}

Keyword: Fermented kitchen waste; Oreochromis niloticus; Relative growth rate; Water additive; Water quality 